\title{
Mensuração e caracterização da dor após episiotomia e sua relação com a limitação de atividades
}

\author{
Measurement and characteristics of pain after episiotomy and its relationship with the activity limitations
} Medición y características del dolor después de la episiotomía y su relación con la limitación de actividades

\author{
Ana Carolina Sartorato Beleza', Cristine Homsi Jorge Ferreira", Ligia de Sousa"l', Ana Márcia Spanó Nakano" \\ ' Universidade Federal de São Paulo, Campus Baixada Santista, Departamento de Ciências da Saúde, \\ Curso de Fisioterapia. Santos-SP, Brasil. \\ "Universidade de São Paulo, Faculdade de Medicina de Ribeirão Preto, \\ Departamento de Biomecânica, Medicina Física e Reabilitação do Aparelho Locomotor. Ribeirão Preto-SP, Brasil. \\ I" Universidade de São Paulo, Escola de Enfermagem de Ribeirão Preto, \\ Programa de Pós-Graduação Enfermagem em Saúde Pública. (Doutoranda) Ribeirão Preto-SP, Brasil. \\ "v Universidade de São Paulo, Escola de Enfermagem de Ribeirão Preto, \\ Programa de Pós-Graduação Enfermagem em Saúde Pública. Ribeirão Preto-SP, Brasil.
}

Submissão: 16-11-2010 Aprovação: 17-05-2012

\section{RESUMO}

Trata-se de um estudo descritivo realizado com cinquenta mulheres em pós-parto vaginal com episiotomia. Objetivou-se mensurar e caracterizar a dor perineal em primíparas submetidas ao parto normal com episiotomia e verificar as atividades limitadas pela dor. Para avaliação da dor foi utilizada a Escala Numérica e o Questionário McGill, bem como um formulário para analisar as atividades que estavam limitadas. A média de dor perineal encontrada foi cinco. As categorias sensorial e avaliação subjetiva foram as mais selecionadas no Questionário McGill. A dor perineal foi caracterizada como latejante, que repuxa, que esquenta, ardida, dolorida, chata, incômoda, que prende e que deixa tensa. Sentar, deitar e deambular foram as atividades mais limitadas. Em conclusão, foi possível verificar a presença de dor perineal nas puérperas causada pela episiotomia e identificar que as atividades de sentar, deitar e deambular estavam limitadas por este sintoma.

Descritores: Dor; Período pós-parto; Episiotomia; Saúde da mulher.

\section{ABSTRACT}

This is a descriptive study, involving fifty women after vaginal delivery with episiotomy. The study aimed to measure and to characterize the perineal pain in primiparae who have had a natural childbirth with episiotomy and to verify the activities limited by the perineal pain. For evaluation of perineal pain, a Numeric Scale and the McGill Pain Questionnaire were used, as well as another questionnaire to analyze the activities limited. In the Numeric Scale, the median of perineal pain referred were five. The sensory and subjective evaluation categories were the most selected in the McGill questionnaire. The perineal pain was characterized as pulsing, pulling, hot, stinging, hurting, annoying, troublesome, tight and tense. The most limited activities were sitting, laying down (onto the bed), and walking. In conclusion, it was possible to verify the presence of perineal pain between women in the postpartum period caused by episiotomy and identify the activities of sitting, lying down and walking were limited for this symptom.

Key words: Pain; Postpartum period; Episiotomy; Women's health.

\section{RESUMEN}

Se realizó un estudio descriptivo de cincuenta mujeres después del parto vaginal con episiotomía. Objetivó-se medir y caracterizar el dolor perineal en primíparas sometidas al parto normal con episiotomía y verificar las actividades limitadas por el dolor perineal. Para la evaluación del dolor se utilizó la Escala Numérica, el Cuestionario de del Dolor de McGill y un cuestionario para analizar las actividades limitadas. En la Escala Numérica, la media de dolor fue cinco. Las categorías de evaluación sensorial y subjetiva fueron los más seleccionados en el cuestionario McGill. La dolor perineal se caracterizó como palpitante, de esfuerzo, que se calienta, quema, duele, aburrido, incómodo, y deja que mantiene estrechos. Sentado, tumbado y caminar son las actividades más limitados. Finalización: fue posible verificar la presencia de dolor perineal entre primíparas causada por la episiotomia e identificar las actividades de sentado, acostado y caminando se vieron limitados por en este síntoma.

Palabras clave: Dolor; Periodo posparto; Episiotomía; Salud de la mujer. 


\section{INTRODUÇÃO}

A dor perineal é descrita pela literatura como sequela frequente do processo de parturição, sendo caracterizada como aguda e considerada a causa mais comum de morbidade no puerpério ${ }^{(1-3)}$. A dor pode levar a manifestações de diversos sintomas, tais como: alterações no padrão de sono, apetite, libido, manifestações de irritabilidade, diminuição da capacidade de concentração e restrições das atividades funcionais, assim como limitação da mobilidade ${ }^{(4)}$. Por outro lado, a ausência desta é capaz de encorajar o retorno precoce às atividades diárias ${ }^{(5)}$.

A saúde biopsicossocial da mulher pode ficar comprometida pela presença da dor. O estresse e o desconforto manifestados no pós-parto muitas vezes não são verbalizados pelas puérperas. A condição dolorosa é pouco valorizada pelas pacientes, pelos familiares e pelos profissionais que as assistem, pois a atenção ao recém-nascido ganha prioridade ${ }^{(1)}$.

A manifestação dolorosa na região perineal pode ocorrer por trauma, especialmente no período expulsivo do parto vaginal. O trauma perineal é definido como a perda da integridade dos tecidos da região genital durante o parto, ocasionado por lesão cirúrgica (episiotomia) ou espontânea(6).

A literatura chama a atenção para a prática da episiotomia e da episiorrafia, que parecem ser os procedimentos que causam maiores desconfortos no pós-parto. Atualmente o uso rotineiro de tal procedimento passa a ser questionado devido às diversas consequências provocadas ao assoalho pélvico feminino, pois a realização desta incisão não garante menores chances de morbidade no puerpério ${ }^{(7-8)}$.

A sensação dolorosa no local da episiotomia pode prejudicar o autocuidado materno e a prestação de cuidados ao recém-nascido. Além disso, pode atrasar a recuperação da mulher, abalar sua autoestima e dificultar o processo de adaptação da mesma ao novo contexto familiar ${ }^{(1,9)}$.

Neste sentido, torna-se necessário conhecer melhor este fenômeno doloroso, uma vez que uma completa e minuciosa avaliação de tal sintoma poderá representar a adoção de terapêuticas mais apropriadas no puerpério. A compreensão do fenômeno doloroso aproxima e melhora a relação terapeuta-paciente, o que pode representar melhores resultados no alívio da queixa dolorosa.

Os profissionais de saúde que atuam na assistência à mulher em processo de parturição e puerpério necessitam do conhecimento a cerca das repercussões que o trauma perineal traz para a saúde materna, como forma de evitar procedimentos desnecessários que causam maior ou menor morbidade materna.

Foram objetivos desta pesquisa os de mensurar e caracterizar a dor perineal em primíparas após parto normal com episiotomia; e verificar as atividades limitadas por este sintoma.

\section{MATERIAIS E MÉTODOS}

Este é um estudo descritivo que foi desenvolvido no Centro de Referência da Saúde da Mulher, situado em Ribeirão Preto, interior do estado de São Paulo. A referida instituição atende exclusivamente gestantes de baixo e médio risco, oriundas do Sistema Único de Saúde.
O pesquisador, ao chegar à maternidade, informava-se com a equipe de plantão sobre pacientes internadas. As mulheres que atendiam aos critérios de inclusão foram informadas do estudo e, após manifestarem a sua vontade em participar, assinaram um termo de consentimento livre e esclarecido. Esta pesquisa foi aprovada pelo Comitê de Ética em Pesquisa da Escola de Enfermagem de Ribeirão Preto/USP (protocolo 0761/2007). Os dados foram coletados entre os meses de juIho e dezembro de 2007.

A população foi composta por 50 mulheres que receberam assistência durante o processo de parturição na referida maternidade. Para cálculo de tamanho da amostra, utilizou-se uma fórmula para "amostra simples" a partir dos escores de dor relatados por meio da escala numérica de 11 pontos e obtidos em teste piloto com oito puérperas. Desse modo, obteve-se uma amostra de 50 sujeitos. Os critérios de inclusão foram: primíparas com gestação de baixo risco, maiores de 18 anos, alfabetizadas (devido à necessidade de compreensão dos instrumentos utilizados no estudo - Escala Numérica e Questionário McGill), em período de 6 a 24 horas de pós-parto vaginal, que não fizeram uso de analgésicos ou anti-inflamatórios e submetidas à episiotomia com episiorrafia.

Para a análise da dor foram utilizados o McGill Pain Questionnaire - MPQ (versão traduzida para o português) ${ }^{(10)}$ e a Escala Numérica Compartimentada em 11 pontos (EN).

O questionário McGill é composto por 4 categorias: sensorial, afetiva, avaliação subjetiva e mista. Cada uma das categorias é dividida em subcategorias: sensorial (10 subcategorias), afetiva (5 subcategorias), avaliação subjetiva (1 subcategoria) e mista (4 subcategorias). Cada subcategoria é composta por um número determinado de palavras que tem como objetivo caracterizar a dor do paciente. Os atributos que melhor representam a dor são aqueles selecionados por, pelo menos, 33\% dos sujeitos da pesquisa, conforme orienta a literatura sobre o tema ${ }^{(10)}$. Além disso, foi analisado o número de palavras escoIhidas dentre os descritores do Questionário McGill, podendo este ser no máximo de 20 palavras.

A dor referida pelas puérperas por meio dos descritores do MPQ e pela EN foi avaliada ao repouso e ao movimento, perguntando à mulher em quais destas situações a dor se manifestava. As puérperas também foram questionadas sobre quais atividades estavam limitadas: sentar, andar, urinar, higiene íntima, evacuação, micção e alimentação ${ }^{(1)}$.

\section{RESULTADOS}

As 50 puérperas participantes deste estudo apresentaram idade média de 22,7 anos e desvio-padrão de 3,9 anos. Concluíram o ensino médio 29 (58\%) das mulheres; 14 (28\%) tinham o ensino médio incompleto, $3(6 \%)$ o ensino fundamental completo e 4 ( $8 \%$ ) o ensino fundamental incompleto.

Todas as mulheres do estudo fizeram acompanhamento pré-natal de suas gestações, com média de 8,1 consultas e desvio padrão de 2,3 consultas. Ao parto, a média da idade gestacional das participantes do estudo definida pela ultrassonografia foi de 39 semanas e desvio-padrão de 1,5 semanas. 
Sobre a assistência obstétrica prestada, foram coletados o número de toques realizados e o tempo de trabalho de parto. A média do número de toques foi de 3,1 toques e desvio-padrão de 1,4 toques. A média da duração do trabalho de parto foi de 199,9 minutos e desvio-padrão de 106,5 minutos. Receberam analgesia durante o trabalho de parto $40(80 \%)$ mulheres. Duas mulheres $(5 \%)$ receberam a raquidiana, 34 $(85 \%)$ receberam a peridural, quatro $(10 \%)$ receberam a analgesia combinada (raqui com peri) e 10 (20\%) não receberam nenhum tipo de analgesia. A média do número de horas pós-parto em que os dados foram coletados foi igual a 16 horas com desvio-padrão de 6 horas.

Os descritores do Questionário McGill que melhor caracterizavam a dor das mulheres foram: latejante (29-58\%), que repuxa $(32-64 \%)$, que esquenta $(24-48 \%)$, ardida $(23-46 \%)$, dolorida (26-52\%), chata (24-48\%), incômoda (27-54\%), que prende (23-46\%) e que deixa tenso (25-50\%). A média do número de palavras escolhidas pelas mulheres foi de 12,42 palavras e desvio-padrão 4,72, sendo o mínimo 5 e o máximo 20.

Ao analisar o nível de dor referido pelas puérperas pela EN, após o parto normal com episiotomia, foi verificado que as mesmas referiram a intensidade de dor 4 (valor mediano), sendo o mínimo 1 e o máximo 10. A média e o desvio-padrão foram de 5 e 2,07, respectivamente.

Referiram presença de dor ao repouso 26 (52\%) puérperas do estudo. Já a dor ao movimento foi referida por $100 \%$ da amostra. As atividades cotidianas referidas pelas mulheres com limitação devido à presença de dor perineal foram: sentar $(n=49,98 \%)$, deitar $(n=45,90 \%)$, deambular $(n=41$, $82 \%)$, micção $(n=20,40 \%)$, evacuação $(n=2,4 \%)$, higiene íntima $(n=22,44 \%)$ e sono $(n=18,36 \%)$.

\section{DISCUSSÃO}

A dor perineal no pós-parto tem sido tema de diversos estudos de pesquisadores no Brasil e no mundo nas últimas décadas, sobretudo no que diz respeito aos fatores de risco que podem levar ao trauma perineal e aos resultados promovidos por recursos de alívio da queixa dolorosa, sendo estes farmacológicos ou não.

Ainda não há consenso entre os autores sobre a influência da idade na avaliação da intensidade dolorosa. Autores referem que as diferenças observadas entre diferentes idades e intensidade de dor, podem ser dependentes do tipo de escala. Ainda, chamam a atenção para o fato de que a maioria dos instrumentos foram validados em amostras de indivíduos jovens, o que poderia influenciar tal avaliação ${ }^{(12)}$.

A idade gestacional das mulheres do estudo foi semelhante. Essa variável foi coletada para que não houvessem diferenças quanto ao tempo de gestação, o que poderia representar mudanças no peso fetal, e consequentemente na distensão do assoalho pélvico no momento do expulsivo ${ }^{(13)}$.

O toque vaginal deve ser reduzido ao número mínimo de vezes. O Manual de Assistência Humanizada à Mulher do Ministério da Saúde indica toques vaginais a cada duas horas a partir do momento em que a parturiente encontra-se na fase ativa do trabalho de parto $^{(14)}$.
No presente estudo a média do número de toques realizados foi igual a 3,84 toques e desvio-padrão de 1,44. A manipulação da região genital pode ocasionar alterações nos tecidos da região, que, se demasiadamente manipulados, podem responder a isto no período puerperal. A mulher pode sofrer com dor e edema; talvez isto possa explicar em parte a ocorrência de dor em mulheres com períneo íntegro após o parto.

A duração do trabalho de parto foi investigada no presente estudo a partir da abertura do partograma da paciente, sendo este em média de 205,58 minutos. O tempo prolongado de trabalho de parto pode representar riscos para região perineal da mulher, bem como para o bem-estar do recém-nascido. Neste sentido, estudos têm sido conduzidos com o objetivo de verificar se o treinamento da musculatura do assoalho pélvico poderia influenciar o tempo total de trabalho de parto e o tempo de período expulsivo ${ }^{(15)}$. O prolongamento destas fases pode representar maior manipulação da região genital da mulher, resultando em queixa de dor no período puerperal.

A analgesia durante o trabalho de parto foi administrada em $80 \%$ (40) das puérperas da presente pesquisa. A analgesia é o principal método farmacológico de alívio da dor utilizado na obstetrícia atual. Contudo, há inúmeras controvérsias acerca do momento adequado para se praticar a analgesia, suas repercussões sobre o feto/recém-nascido e se há aumento de práticas intervencionistas com seu uso.

Encontra-se na literatura um estudo comparativo entre a técnica peridural contínua com a combinada, de que participaram 40 parturientes, divididas em dois grupos, de forma aleatória: o grupo 1 recebeu analgesia peridural contínua e o grupo 2 recebeu a combinada. Cerca de 30\% (12) dos partos foram fórceps e 12,5\% (5) foram cesárea. Os autores justificaram o número alto de fórceps como uma conduta obstétrica do hospital onde o estudo foi realizado ${ }^{(16)}$.

Uma revisão sistemática sobre os efeitos de todas as modalidades de analgesia para a mãe e para o recém-nascido concluiu que a analgesia epidural é eficaz no alívio da dor do trabalho de parto, porém as mulheres sofrem maior risco de partos instrumentalizados (realização de episiotomia e parto fórceps) o que aumenta a chance do trauma perineal ${ }^{(17)}$.

Quanto à avaliação da intensidade da dor, realizada por meio da escala numérica neste estudo, mostrou média e desvio padrão iguais a 5 e 2,07. Outro estudo avaliou a dor na região perineal após o parto vaginal com episiotomia e encontrou média de 4,2 e desvio-padrão de $2^{(18)}$.

Mais da metade das mulheres do presente estudo (26-52\%) queixaram-se de dor ao repouso. Tal fato reforça que este sintoma pode limitar a puérpera em diversas funções e que mesmo em repouso o alívio da queixa não ocorre. Algumas atividades cotidianas podem estar limitadas pela presença de dor durante os movimentos. Neste sentido, também foram investigadas as atividades cotidianas realizadas pela mulher no período puerperal dentro da maternidade, que poderiam estar limitadas em virtude da presença da dor.

Sentar, deitar e deambular foram as atividades mais limitadas referidas pelas mulheres em ambos os grupos, decorrente do sintoma doloroso. Outros autores também questionaram essas limitações para puérperas no primeiro dia pós-parto e 
encontraram que, entre 97 mulheres com episiotomia, 12,37\% referiram dor para sentar, e $7,21 \%$ ainda continuava com dor nesta atividade sete dias após o parto ${ }^{(2)}$. Em outro estudo, a autora encontrou que a dor estava presente ao sentar em $62,5 \%$ de uma amostra de 45 mulheres $^{(18)}$.

$\mathrm{Na}$ presente pesquisa as mulheres não referiram dor ao evacuar, por ainda não terem realizado esta atividade. Algumas mulheres referiram medo de "romper os pontos". Deve-se considerar, neste momento, o próprio processo fisiológico do puerpério. A primeira evacuação pode se retardar, devido às alterações causadas na musculatura lisa do intestino, que sofre ação do hormônio progesterona.

Neste estudo, todas as mulheres que fizeram parte da amostra eram primíparas. Este critério foi assim determinado, uma vez que a experiência dolorosa atual poderia ser influenciada por experiências anteriores vividas pelos sujeitos. Acredita-se que uma experiência anterior de parto vaginal com episiotomia poderia alterar a avaliação da intensidade da dor relatada pela puérpera.

Em relação à paridade, autores ${ }^{(19)}$ estudaram a taxa de episiotomia e sua associação com dor perineal nos primeiros dois meses após o parto. Trinta e um porcento das primíparas do estudo foram submetidas à episiotomia, das quais $82 \%$ apresentaram queixa de dor perineal. Quanto às multíparas, a episiotomia foi realizada em $19 \%$, e destas, apenas $18 \%$ relataram dor penineal dois meses após o nascimento. Entretanto, a queixa de dor entre as multíparas submetidas à episiotomia foi três vezes maior que aquelas que não foram submetidas à episiotomia.

É possível entender que, a percepção dolorosa das multíparas é menor que das primíparas em relação á dor perineal, porém, a sensação dolorosa é ainda maior do que em mulheres que não realizaram a episiotomia.

A frequência da dor perineal após o parto normal e a associação entre trauma e dor nesta mesma região foi objeto de investigação de dois pesquisadores. Foram selecionadas para estudo 444 mulheres, incluindo puérperas com períneo íntegro, $1^{\circ}, 2^{\circ}, 3^{\circ}$ e $4^{\circ}$ graus de laceração e episiotomia. Os autores encontraram que primíparas sofreram traumas na região genital com maior frequência, mais partos instrumentais e receberam mais analgesia epidural no segundo estágio(2). Os escores de dor avaliados pelo Questionário McGill foram menores nas multíparas. Foi destacado que, quanto maior o trauma perineal, maior o escore de dor, maior escolha por palavras severas para descreverem a queixa e maior o uso de analgésicos no grupo que sofreu laceração ou episiotomia. No presente estudo é possível observar que a presença de dor foi manifestada por mais da metade das puérperas com episiotomia analisadas durante repouso e, por todas, durante os movimentos ${ }^{(2)}$.

Outro estudo, envolvendo 143 mulheres, encontrou 47,5\% de episiotomia, 19,6\% de lacerações de primeiro ou segundo grau e 32,9\% de períneo íntegro. Estes autores concluíram que a episiotomia causou duas vezes mais queixas de dor perineal do que o grupo com períneo íntegro, destacando o incômodo gerado pelas altas taxas de episiotomia ${ }^{(20)}$.

Existem achados na literatura em que a dor perineal é maior em mulheres que sofreram lacerações de primeiro, segundo, terceiro e quarto graus ou episiotomia que aquelas que tiveram o períneo intacto após um dia, cinco dias ou sete semanas do nascimento. Estes mesmos autores compararam a dor perineal entre mulheres submetidas à episiotomia e que sofreram laceração de segundo grau, sendo que, cinco dias após o parto a episiotomia gerou significativamente mais dor para as mulheres em repouso e ao sentar-se que a laceração de segundo grau ${ }^{(21)}$.

A presença de grandes traumas ou episiotomia, além de causarem maior queixa de dor entre as mulheres após o nascimento, propiciam um maior uso de medicamentos para alívio de dor. Há relatos de maiores escores de dor perineal entre mulheres com grandes traumas na região perineal, sendo que $76,3 \%$ receberam medicamentos (ibuprofeno ou paracetamol) para alívio da queixa ${ }^{(20)}$.

A dor foi caracterizada pelas mulheres deste estudo como latejante, que repuxa, que esquenta, ardida, dolorida, chata, incômoda, que prende e que deixa tenso. É possível encontrar na literatura um outro estudo que avaliou a dor perineal no período pós-parto vaginal com episiotomia também por meio do MPQ. Os autores encontraram que os descritores que melhor caracterizaram a dor foram: dolorida; que repuxa; incômoda; chata; ardida; pica como uma agulhada; latejante; em pressão. ${ }^{(23)}$

A análise do número de palavras escolhidas foi semelhante entre as categorias. Vale destacar que a categoria avaliação subjetiva foi selecionada por todas as $50(100 \%)$ puérperas.

As puérperas deste estudo escolheram nove palavras para caracterizar a dor perineal no pós-parto vaginal com episiotomia. Outros autores ${ }^{(18)}$ encontraram o mesmo número de palavras para caracterizar a dor perineal após o parto, o que corrobora os achados da presente pesquisa.

Não foram encontrados, até o momento, demais estudos na língua portuguesa para que possam ser comparados os dados encontrados neste. Os dados obtidos pelo MPQ tornam-se um pouco limitados para a comparação com outras pesquisas, dadas as diferenças de línguas. Por esta razão é que as escalas unidimensionais têm sido mais utilizadas nas pesquisas relacionadas à dor ${ }^{(11)}$.

São necessários estudos nacionais que façam o seguimento da puérpera ao longo do puerpério tardio. Deve-se investigar a frequência de dor fora do ambiente hospitalar, as queixas relacionadas à função sexual e a ocorrência de incontinência urinária e/ou fecal, uma vez que a literatura internacional mostra o prejuízo que tais morbidades trazem à mulher.

Outra limitação do estudo foi a falta de um grupo de puérperas com períneo íntegro ou lacerações perineais espontâneas para comparar os escores de dor e identificar os desconfortos por ela produzidos. Assim, mais estudos são necessários nesta área do conhecimento, pois o período puerperal é pouco discutido na literatura mundial.

Vale destacar que os resultados do presente estudo aplicam-se à amostra estudada em uma maternidade do interior do Estado de São Paulo e que atende clientela proveniente do Sistema Único de Saúde.

\section{CONCLUSÃO}

A dor perineal após episiotomia foi referida pelas puérperas do estudo nas primeiras 24 horas pós-parto. A queixa dolorosa foi avaliada por meio da Escala Numérica Compartimentada 
em 11 pontos, sendo encontrada a mediana de 4. Pelos descritores do Questionário McGill, a dor perineal pós-parto vaginal com episiotomia foi caracterizada pelas puérperas como latejante, que repuxa, que esquenta, ardida, dolorida, chata, incômoda, que prende e que deixa tenso. As puérperas referiram sentir dor mesmo em repouso. As atividades que mais estavam limitadas pela dor na região perineal foram sentar, deitar e deambular. Todas as puérperas do estudo relataram dor durante o movimento.

É importante destacar que a presença da dor e das limitações por ela causada durante o período pós-parto é pouco valorizada entre os profissionais da saúde, que negligenciam os cuidados maternos em detrimento dos cuidados neonatais. Tal acontecimento pode afetar a qualidade de vida da puérpera, pois as limitações sentidas em decorrência da dor interferem na sua mobilidade.

A partir do conhecimento das repercussões da dor no cotidiano da puérpera, será possível investigar a utilização de recursos não farmacológicos para alívio desta queixa. Conforme exposto, a dor perineal limita as atividades da mulher no período pós-parto, o que pode refletir em dificuldades no autocuidado e no cuidado ao recém-nascido.

\section{REFERÊNCIAS}

1. Alexandre CW. Prevalência, características e impacto da dor no cotidiano da puérpera. São Paulo. Dissertação [Mestrado em Enfermagem]- Universidade de São Paulo; 2002.

2. Macarthur AJ, Macarthur C. Incidence, severity, and determinants of perineal pain after vaginal delivery: a prospective cohort study. Am J Obstet Gynecol 2004;191:1199-204.

3. Riesco MLG, Costa, ASC, Almeida, SFS, Basile, ALO, Oliveira, SMJV. Episiotomia, laceração e integridade perineal em partos normais: análise de fatores associados. Rev Enferm UERJ 2011;(19);77-83.

4. Kreling MCGD, Cruz DALM, Pimenta CAM. Prevalência da dor crônica em adultos. Rev Bras Enferm 2006;(59):509-13.

5. Imarengiaye $\mathrm{CO}$, Andet $\mathrm{AB}$. Postpartum perineal pain among Nigerian women. West Afr J Med 2008; 27:148-51.

6. Johanson R. Perineal massage for prevention of perineal trauma in childbirth. Lancet 2000;335:250-1.

7. Carroli GB. Episiotomy for vaginal birth. Cochrane [Homepage na internet]. 1999 [acesso em 18 maio 2008]; Disponível em http://cochrane.bvsalud.org/cochrane/ main.php?lib $=C O C \&$ searchExp $=$ episiotomia\&lang $=p t$

8. Fritel X, Schaal JP, Bertrand V, Levet C, Pigné A. Pelvic Floor disorders 4 years after delivery: a comparison study of restrictive versus systematic episiotomy. B J Obstet Gynecol 2008;115:247-52.

9. Andrews $\mathrm{V}$, Thakar R, Sultan AH, Jones PW. Evaluation of postpartum perineal pain and dyspareunia-A prospective study. Eur J Obst Gynecol Reprod Biol 2008;137:152-6.

10. Castro CES. A formulação linguística da dor: versão brasileira do Questionário McGill de dor. São Carlos. Dissertação [Mestrado em Fisioterapia]- Universidade Federal de São Carlos; 1999.

11. Santana JM. Efeito hipoalgésico da estimulação nervosa transcutânea (TENS) em modelo de dor pós-operatória visceral. Ribeirão Preto. Tese [Doutorado em Ciências da Reabilitação]- Faculdade de Medicina de Ribeirão Preto, Universidade de São Paulo; 2006.

12. Gagliesi L, Melzack, R. Age differences in the quality of chronic pain: a preliminary study. Pain Res Manage 1997;2:157-162.
13. Scarabotto LB, Riesco, MLG. Fatores relacionados ao trauma perineal no parto normal em mulheres nulíparas. Rev Esc Enferm USP 2006;(40):389-96.

14. Ministério da Saúde (Brasil) Parto, Aborto e puerpério: assistência humanizada a mulher. Secretaria de Políticas de Saúde; Área Técnica de Saúde da Mulher. Brasília; 2001.

15. Dias Letícia A. R., Driusso Patricia, Aita Daniella L. C. C., Quintana Silvana M., Bø Kari, Ferreira Cristine H. J.. Effect of pelvic floor muscle training on labour and newborn outcomes: a randomized controlled trial. Rev Bras Fisioter 2011;15:487-93.

16. Côrtes CAF, Sanchez CA, Oliveira AS, Sanchez FM. Analgesia de parto: estudo comparativo entre anestesia combinada raquiperidural versus anestesia peridural contínua. Rev Bras Anestesiol 2007;(57): 39-51.

17. Anim-Somuah M, Smyth R, Howell C. Epidural versus non-epidural or no analgesia in labour. Intervent Rev [periódico na internet]. [acesso em 01 jun 2008]; Disponível em: http://mrw.interscience.wiley.com/cochrane/clsysrev/ articles/CD000331/frame.html

18. Pitangui ACR, Sousa L, Ferreira CHJ, Gomes FA, Nakano AMS. Mensuração e características da dor perineal em primíparas submetidas à episiotomia. Acta Paul Enferm 2009;(22):77-82.

19. Declercq E, Cunningham DK, Johnson C, Sakala C. Mothers' reports of postpartum pain associated with vaginal and cesarean deliveries: results of a national survey. Birth 2008;35:16-24.

20. Imarengiaye $\mathrm{CO}$, Andet $\mathrm{AB}$. Postpartum perineal pain among Nigerian women. West Afr J Med 2008; 27:148-51.

21. Andrews $V$, Thakar R, Sultan $A H$, Jones PW. Evaluation of postpartum perineal pain and dyspareunia-A prospective study. Eur J Obst Gynecol Reprod Biol 2008;137:152-6.

22. Leeman L, Fullilove AM, Borders N, Manocchio R, Albers LL, Rogers RG. Postpartum Perineal Pain in a Low Episiotomy Setting: Association with Severity of Genital Trauma, Labor Care, and Birth Variables. Birth 2009;36:283-8.

23. Pintagui AC. Avaliação do efeito da estimulação elétrica nervosa transcutânea (TENS) no alívio da dor pós-episiotomia em primíparas submetidas ao parto normal. Ribeirão Preto. Dissertação [Mestrado em Enfermagem]- Escola de Enfermagem de Ribeirão Preto, Universidade de São Paulo; 2007. 